\title{
Model Independent Diagnostics in Interacting Dark Energy Models
}

\author{
Ying Jiang ${ }^{1}$, Zhongxu Han ${ }^{1}$, Qian Zhang ${ }^{2}$, Weiqiang Yang ${ }^{1}$, Yabo $\mathrm{Wu}^{1, *}$, Jinyang $\mathrm{Li}^{1}$, \\ Han Lou ${ }^{1}$, Chenchen Zhao ${ }^{1}$ and Yan Wang ${ }^{1}$ \\ 1 Department of Physics, Liaoning Normal University, Dalian 116029, China; jy18242067301@163.com (Y.J.); \\ h17866582056@163.com (Z.H.); wqyang@lnnu.edu.cn (W.Y.); li15566528001@163.com (J.L.); \\ lh1106384639@163.com (H.L.); zcc922zcc@163.com (C.Z.); wangyan551129@126.com (Y.W.) \\ 2 Department of Science, Shenyang University of Chemical Technology, Shenyang 110142, China; \\ zhangqian030128@syuct.edu.cn \\ * Correspondence: ybwu61@126.com
}

Received: 24 February 2020; Accepted: 25 March 2020; Published: 30 March 2020

\begin{abstract}
In this paper, we have explored non-gravitational interaction dark energy model between dark energy and cold dark matter (CDM). The main purpose of this work is to explore the deviations from $\Lambda C D M$ and to distinguish the evolution trajectory of the interaction dark energy model with different parameters. Six forms of interaction were focused on. We have analyzed the deceleration parameters of the coupled model and explored the theoretical models through statefinder hierarchy and the $\mathrm{Om}$ diagnostic. We have considered that the Equation of State (EoS) parameter $w$ and parameter $\xi$ take different values on the interaction dark energy model respectively and keep another parameter unchanged. It was found that $q_{(z)}$ is the worst of the four diagnoses. Om, $S_{3}^{(1)}$ and $S_{4}^{(1)}$ are good tools to distinguish the interaction dark energy model from $\Lambda C D M . S_{3}^{(1)}$ is a better diagnostic tool when the state parameter $w$ changes and the coupling parameter $\xi$ is fixed, while $S_{4}^{(1)}$ works better when the coupling parameter $\xi$ changes and the state parameter $w$ is fixed.
\end{abstract}

Keywords: dark energy; interaction; diagnostic

\section{Introduction}

Cosmology is one of the most active research areas. It is well known that modern cosmology was established and developed with the birth of general relativity [1,2]. Therefore, general relativity plays a very important role in the study of gravity and cosmology, and so far can be verified by many experiments without adding special case assumptions. Even so, there is evidence that the theory is incomplete-the search for quantum gravity and the reality of space-time singularities remain to be solved; the results of experimental observations supporting the existence of dark matter and dark energy imply a desire for new physics. However, general relativity is still full of possibilities that deserve deep exploration-mathematical relativists are seeking to understand the nature of singularities and the basic properties of the Einstein field equation; and, after the first direct observation of gravitational waves in 2016, subsequent contests and development applications are continuing, human beings hope to be able to create more opportunities to test the theory's validity in a field of gravity that is much stronger than it has ever been. So today, a hundred years after Einstein published his theory, general relativity is still a highly active research field.

After the establishment of modern cosmology with the advent of general relativity, scientists became interested in the causes of the universe's accelerating expansion. In recent years, astronomical observations have shown that our universe is undergoing accelerated expansion [3-7]. Many people are trying to find a perfect explanation for the accelerating expansion of the universe. The most likely 
explanation is that the universe is currently being dominated by an exotic component with negative pressure, named dark energy (DE). According to Planck 2015 observational results [8], the universe is spatially flat, and it consists of about 68.4 percent dark energy, 26.7 percent dark matter, 4.9 percent baryon matter.

As scientists study the accelerating expansion of the universe, various theoretical models have been proposed. Among them, the cosmological constant model ( $\Lambda C D M$ model) is the simplest and most idealized of the many models that explain today's accelerating expansion of the universe [9-14], its state parameter is $w_{\Lambda}=-1$. This cosmological constant was originally introduced by Einstein into a static cosmological model. From 1915 to 1917, Einstein explained our universe with the general relativity gravitational field equation, trying to build a new cosmological model. At that time, the scientific community still thought that the universe was still and did not change with time, so Einstein added the cosmological constant to get a stable solution. The function of this constant in Einstein's equation was repulsion, so one possibility of dark energy was the cosmological constant. Einstein gave up the constant and thought it was a mistake after Hubble discovered that the universe accelerated expansion in 1929. However, the cosmological constant was reintroduced in 1998 after the discovery of the accelerating expansion of the universe by the observed type 1a supernova. One finds that the cosmological constant model ( $\Lambda C D M$ model)is just enough to explain that the expansion of the universe is due to repulsion of a component. This model is very consistent with astronomical observations, there are still some problems with the $\Lambda C D M$ model, such as fine-tuning and cosmic coincidence.

To alleviate these problems, people have proposed dynamic dark energy model one after another: quintessence [15,16], phantom [17], quintom [18,19], holographic dark energy model [20-24], Chaplygin gas (CG) model [25-27], Dirac-Born-Infeld (DBI) dark energy [28,29], and so forth.

In this paper, we have studied the non-gravitational interaction dark energy model between dark energy and cold dark matter in which the equation of state (EoS) parameter $w$ is a constant, this kind of interacting dark energy model have been widely studied [30-55], and in this paper the coupled dark energy model is called IwCDM model. It can also explain the accelerated expansion of the large scale universe [56-65]. Meanwhile, the existence of non-gravitational interaction not only lead to an alleviation of the coincidence problem, but also make theoretical models work better. Compared to $\Lambda C D M$ or its extended model $w C D M$, the the interacting dark energy model make dark energy and dark matter with energy exchange from the background evolution, in this way in the early time of expanding universe, the less dark energy will implicitly affect the evolution of dark matter, it will slightly influence the matter structure growth, meanwhile in the late time of expanding universe, the dark matter will exchange the energy with dark energy, the interaction will affect the universe acceleration. In this paper, we will focus on the background evolution of interacting dark energy model. In addition, we also have explored the deviation of the interaction dark energy model from $\Lambda C D M$ and the behavior of evolution trajectory when the interaction dark energy model takes different parameter values through the Statefinder hierarchy [56,66-74] and Om [75-77] diagnostic. The state finder hierarchy is a sensitive diagnostic of DE, which uses the higher-order derivatives of $a(t)$ and can break the degeneracy of the model very well. However, the advantage of the Om diagnostic is its less dependency on the matter density. That means the Om diagnostic is much easier than the statefinder hierarchy.

In the spatially homogeneous and isotropic Friedmann-Robertson-Walker (FRW) universe filled with DE (de), CDM (c), baryons (b), and radiation (r). The continuity equation for each component can be written as follows:

$$
\begin{aligned}
\rho_{d e}+3 H(1+w) \rho_{d e} & =-Q, \\
\dot{\rho}_{c}+3 H \rho_{c} & =Q, \\
\dot{\rho}_{b}+3 H \rho_{b} & =0 \\
\dot{\rho}_{r}+4 H \rho_{r} & =0
\end{aligned}
$$


where the dot denotes a derivative with respect to time $t, H=\dot{a} / a$ is the Hubble parameter, $\rho_{i}$ is the energy density of each component, for $i=d e, c, b$, and $r$ respectively, and $Q$ is the energy transfer rate between DE and CDM. In this paper, six interacting forms were considered: $Q_{1}=\xi H \frac{\rho_{c} \rho_{d e}}{\rho_{c}+\rho_{d e}}$, $Q_{2}=\xi H \rho_{c}, Q_{3}=\xi H \sqrt{\rho_{c} \rho_{d e}}, Q_{4}=\xi H(1+w) \rho_{c}, Q_{5}=\xi H(1+w) \frac{\rho_{c} \rho_{d e}}{\rho_{c}+\rho_{d e}}, Q_{6}=\xi H(1+w) \sqrt{\rho_{c} \rho_{d e}}$, where $\xi$ is the dimensionless coupling parameter. For these six interaction terms, there are some relations, such as $Q_{1}$ and $Q_{5}, Q_{2}$ and $Q_{4}, Q_{3}$ and $Q_{6}$, here we show the evolutional trajectories of dimensionless density parameter in Figure 1.
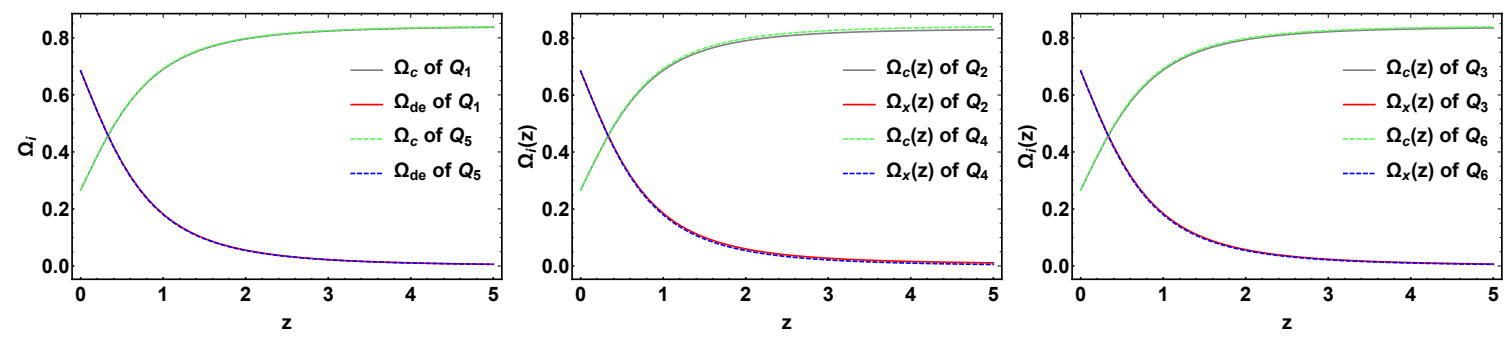

Figure 1. The evolution trajectories of $\Omega_{i}$ versus red shift $\mathrm{z}$ of interacting dark energy models, to compare $Q_{1}$ and $Q_{5}, Q_{2}$ and $Q_{4}, Q_{3}$ and $Q_{6}$, here we fixed $w=-1.1$ and $\xi=0.02$, the other related parameters was fixed as the results of Planck 2015, $\Omega_{d e 0}=0.684, \Omega_{c 0}=0.267, \Omega_{b 0}=0.049$, $\Omega_{r 0}=0.0001$.

The paper is organized as follows. In Section 2, two diagnostic methods were briefly introduced-the Statefinder hierarchy and the Om diagnostic. In Section 3, we have explored the evolutionary behavior of the interacting dark energy model through the diagnostic tools-the deceleration parameter $q$, the Statefinder hierarchy, the Om diagnostic, and compared them $[9,10,72,78]$. Section 4 is the summary of this paper.

\section{Statefinder Hierarchy and Om Diagnostic}

Since many dark energy models are pretty good explanations for the accelerating expansion of the universe, in order to distinguish between them, we need to use some distinguishing tools. So various tools have been developed to distinguish dark energy models. In this section, we adopted the Statefinder hierarchy diagnostic and Om diagnostic.

\subsection{The Statefinder Hierarchy}

The scale factor of the Universe can be Taylor expanded around the present epoch $t_{0}$ as follows [66]

$$
\frac{a(t)}{a_{0}}=1+\sum \frac{A_{n}\left(t_{0}\right)}{n !}\left[H_{0}\left(t-t_{0}\right)\right]^{n}
$$

where $A_{n}=\frac{a^{(n)}}{a H^{n}}, n \in N, a^{(n)}$ is the $n$th derivative of the scale factor with respect to time, and $q \equiv-A_{2}$ is the deceleration parameter. For $\Lambda C D M$,

$$
\begin{aligned}
& A_{2}=1-\frac{3}{2} \Omega_{m} \\
& A_{3}=1 \\
& A_{4}=1-\frac{9}{2} \Omega_{m} \\
& A_{5}=1+3 \Omega_{m}+\frac{27}{2} \Omega_{m}^{2}, \quad \text { etc. }
\end{aligned}
$$


where $\Omega_{m}=\frac{2}{3}(1+q), A_{n}(n \geq 3)$ is transformed to statefnder hierarchy, $S_{n}^{(1)}$, which can be defined as [66]:

$$
\begin{aligned}
& S_{3}^{(1)}=A_{3}, \\
& S_{4}^{(1)}=A_{4}+3(1+q), \\
& S_{5}^{(1)}=A_{5}-2(4+3 q)(1+q), \quad \text { etc. }
\end{aligned}
$$

obviously, $S_{n}^{(1)}$ of the $\Lambda C D M$ model is a fixed value during the cosmic expansion, $S_{n \mid \Lambda C D M}^{(1)}=1$. However, the $S_{n}^{(1)}$ value of other DE models is not fixed, so we can easily distinguish the $\Lambda C D M$ model from other DE models through this diagnostic method. Now, we consider a spatially FRW universe containing DE, CDM, baryons, and radiation, $S_{n \mid \Lambda C D M}^{(1)}$ is no longer a fixed value, but changes slowly over time during the expansion of the universe. However, $\Lambda C D M$ can also be regarded as a reference model.

In this paper, we have discussed the interaction DE model, so $S_{3}^{(1)}$ and $S_{4}^{(1)}$ can be represented as follows [56]:

$$
\begin{aligned}
S_{3}^{(1)}=\quad & 1+\frac{9}{2} \Omega_{d e} w+\frac{9}{2} \Omega_{d e} w^{2}-\frac{3}{2} \Omega_{d e} w^{\prime}+2 \Omega_{r}+\frac{3 w Q}{2 H \rho}, \\
S_{4}^{(1)}=\quad & 1-\frac{9}{4} w \Omega_{d e}^{2}\left(3 w+3 w^{2}-w^{\prime}\right)-\frac{3}{3} \Omega_{d e}[w(21+ \\
& \left.39 w+18 w^{2}\right)+\Omega_{r}\left(7 w+3 w^{2}-w^{\prime}\right)-(13+ \\
& \left.18 w) w^{\prime}+w^{\prime \prime}\right]-\Omega_{r}\left(9+\Omega_{r}\right)-\frac{3 w Q}{2 H \rho}(2+3 w) \\
& +\frac{3 w Q^{\prime}}{2 H \rho}+\frac{3 w^{\prime} Q}{H \rho},
\end{aligned}
$$

where the prime denotes the derivative with respect to $x=\ln a$ and $\rho=\Sigma \rho_{i}$. Equations (13) and (14) can be applied to all the interaction DE models. When we take different forms of interaction, the corresponding physical quantities can be substituted into the above formulas respectively, also the corresponding $S_{n}^{(1)}$ value can be obtained.

\subsection{The Om Diagnostic}

In this section, another commonly used method to distinguish dark energy models will be introduced, namely $\mathrm{Om}$ diagnosis. The $\mathrm{Om}$ diagnostic is based on Hubble parameters, it can provide a null diagnostic of the concordance cosmology $(\Lambda C D M)$ and distinguish between models without reference to the density of matter. The $O m$ diagnostic can be defined as [75]:

$$
O m(x)=\frac{h^{2}(x)-1}{x^{3}-1}
$$

where $h(x)=H(x) / H_{0}$ and $x=1+z . H(x)$ is the Hubble parameter and $H_{0}$ is the value of the Hubble parameter at $z=0$. For $\Lambda C D M$, we have found that $\operatorname{Om}(x)=\Omega_{0 d m}$.

\section{Exploration of Interacting Dark Energy with Statefinder Hierarchy and Om Diagnostic}

In this section, we shall discuss the deviations from $\Lambda C D M$ and distinguish the evolution trajectory of the interaction dark energy model with different parameters by using the diagnostics of statefinder hierarchy and the $\mathrm{Om}$ diagnostic. Here, we only consider the influence of EoS parameter $w$ and coupling parameter $\xi$. In addition, for all models, the present-day fractional density parameters of DE, Cold Dark Matter (CDM), baryons, and radiation are fixed to be, according to Planck 2015 observational results [8], $\Omega_{d e 0}=0.684, \Omega_{c 0}=0.267, \Omega_{b 0}=0.049, \Omega_{r 0}=0.0001$. Firstly, we shall 
consider the influence of the EoS parameter $w$ on IwCDM model and keep the coupling parameter $\xi$ unchanged. Then, we will discuss the influence of the coupling parameter $\xi$ on IwCDM model, and the EoS parameter $w$ will be kept unchanged.

\subsection{Consider the Change of $w$ and Keep the Parameter $\xi$ Constant}

In this subsection we only consider the EoS parameter $w$ to take different values on the interaction dark energy model, and keep the parameter $\xi$ unchanged. Here we have chosen $\xi=0.02$, and let the parameters of $w$ be equal to $-0.9,-1.1$, and -1.2 , respectively.

First, the IwCDM models with $Q_{1}$ (denoted as IwCDM1) has been diagnosed according to the deceleration parameter $q$, the statefinder hierarchy parameter $S_{3}^{(1)}, S_{4}^{(1)}$ and the Om diagnostic respectively. We have plotted the function $S_{3}^{(1)}, S_{4}^{(1)}, q$ and Om versus red shift $z$ for the IwCDM1 model in Figure 2. Here we have chosen $\xi=0.02, w=-0.9,-1.1$ and -1.2 .

In Figure 2, we have seen that for $q(z)$, the change of parameter $w$ has little effect on the transition red shift, and in the high red shift region, there is a high degree of degeneracy between the evolution trajectories of the model with different parameter values and even including $\Lambda C D M$. However, in the low red shift region the degeneracy is destroyed to some extent. But these evolutionary trajectories, including $\Lambda C D M$, are very close to each other. Therefore, it can be concluded that the deceleration parameter $q$ does not distinguish the model very well. And from the image of the deceleration parameters, we can see that the evolution of the universe is from deceleration expansion to accelerated expansion. For $S_{3}^{(1)}$, the degeneracy is perfectly broken in the low red shift region, and because the parameters $w$ are different, the curve shows the trend of symmetry. In the high red shift region, the curve of the model is somewhat degeneracy. But it doesn't matter, because the observations are mainly concentrated in the low red shift range $(z \leq 1)$. Also, it is easy to separate $\Lambda C D M$ from IwCDM1. For $S_{4}^{(1)}$, we have seen that in the high red shift region, the evolution trajectory of different parameters is highly degenerate, and it's hard to distinguish between $\Lambda C D M$ and IwCDM1. In the low red shift region, the evolution curves of different parameters $w$ are hugely separate from each other, yet there is a bit of degeneracy between the lines of $w=-0.9$ and $\Lambda C D M$. We still think $S_{4}^{(1)}$ is a good tool to distinguish between $\Lambda C D M$ from I $w C D M 1$, but $S_{3}^{(1)}$ works better. For Om, interestingly, the evolution trend of the IwCDM1 model is similar to that in the $S_{3}^{(1)}$, the three curves in the low red-shift region are all separated from each other and can be well distinguished from the $\Lambda C D M$, except that the distance between them is obviously not far from the $S_{3}^{(1)}$. Overall, the evolution trajectories corresponding to different parameters are separated further in the $S_{3}^{(1)}$, and $S_{3}^{(1)}$ can better distinguish $\Lambda C D M$ from I $w C D M 1$, in the low red shift region. From this point of view, the diagnostic effect of $S_{3}^{(1)}$ is the best.

In Figure 3, we have been discussed the model with interaction $Q_{2}$ (denoted as IwCDM2), as well as made a comparison with the $\Lambda C D M$ model and explored degeneracy between the evolution trajectories of the model with different parameter values. We have seen that the four diagnostic curves of IwCDM2 model are similar to those of IwCDM1, and $S_{3}^{(1)}, S_{4}^{(1)}, \mathrm{Om}$ also can distinguish between $\Lambda C D M$ and ICDM2. Similarly among them, $S_{3}^{(1)}$ performs better.

In Figure 4, the evolutionary trajectories are similar to those of the first two models, and will not be elaborated on here. From the images of the above three interaction models, it can be concluded that $S_{3}^{(1)}$ has a better effect on distinguishing the curves with different parameters of the model itself, and $S_{3}^{(1)}$ is also better in distinguishing $\Lambda C D M$ and ICDM2.

Next, we will discuss the three models with interaction terms $Q_{4}, Q_{5}$ and $Q_{6}$, which are called IwCDM4, IwCDM5, IwCDM6. The interactions of these three models are actually multiplied by $(1+w)$ on the basis of $Q_{1}, Q_{2}$ and $Q_{3}$, other rules are similar to the first three models. We have draw a conclusion from Figures $5-7, S_{3}^{(1)}, S_{4}^{(1)}$ and $O m$ diagnostic methods all can distinguish different model well in the low red shift region. For $q_{(z)}$, the curve has a large degeneracy in the low red shift region. 
In the same light, $S_{3}^{(1)}$ is the best of the four diagnostic tools. From the above discussion, we has been found that $q_{(z)}$ is the worst of the four diagnoses, with the change of state parameters, the evolution trajectory in $q$ is not greatly affected. $\mathrm{Om}, S_{3}^{(1)}$ and $S_{4}^{(1)}$ are all good diagnostic tools to distinguish different dark energy models. By comparison, $S_{3}^{(1)}$ is the best tool for diagnostic results for these interactive dark energy models.
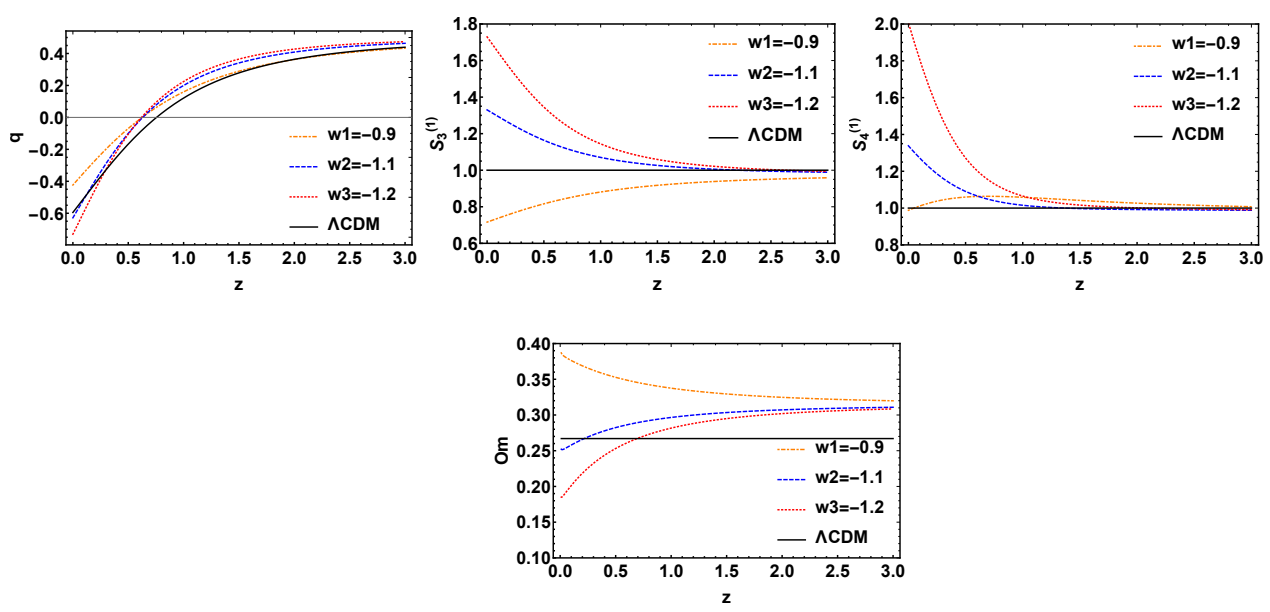

Figure 2. The evolution trajectories of $q, S_{3}^{(1)}, S_{4}^{(1)}$ and $O m$, respectively, versus red shift $\mathrm{z}$ for the $I w C D M 1$ model with variable $w$ and fixed $\xi=0.02$. The evolution curve of $\Lambda C D M$ is also shown in these sets of figures.
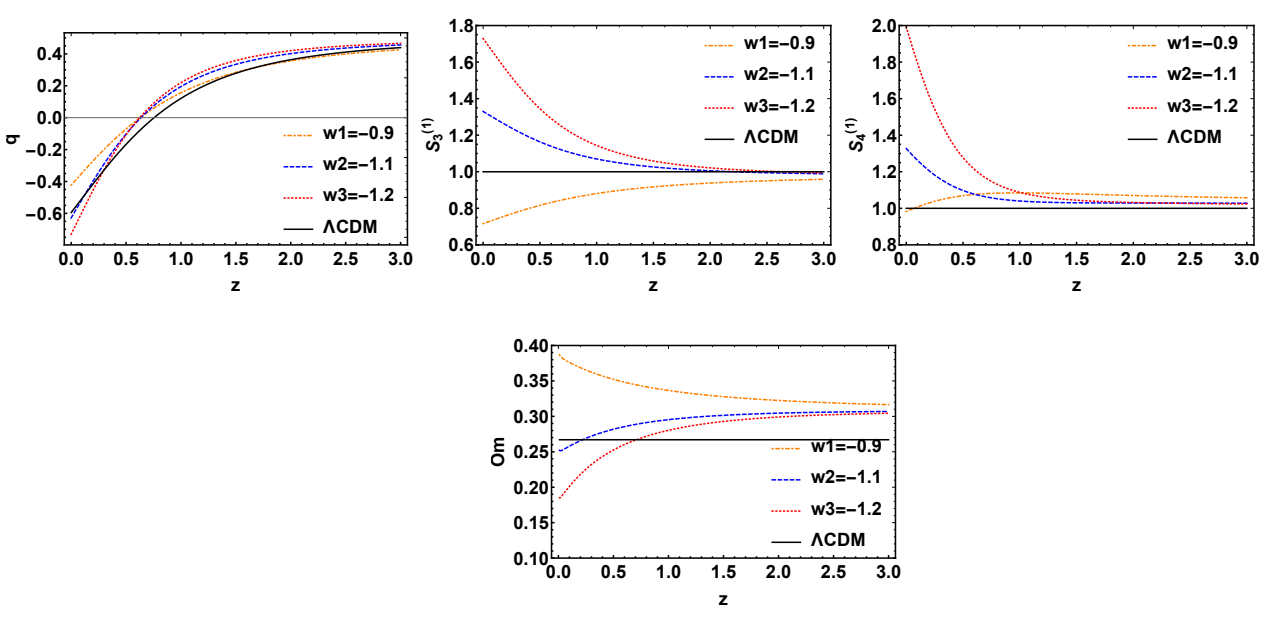

Figure 3. The evolution trajectories of $q, S_{3}^{(1)}, S_{4}^{(1)}$ and $O m$, respectively, versus red shift $\mathrm{z}$ for the $I w C D M 2$ model with variable $w$ and fixed $\xi=0.02$. The evolution curve of $\Lambda C D M$ is also shown in these sets of figures. 

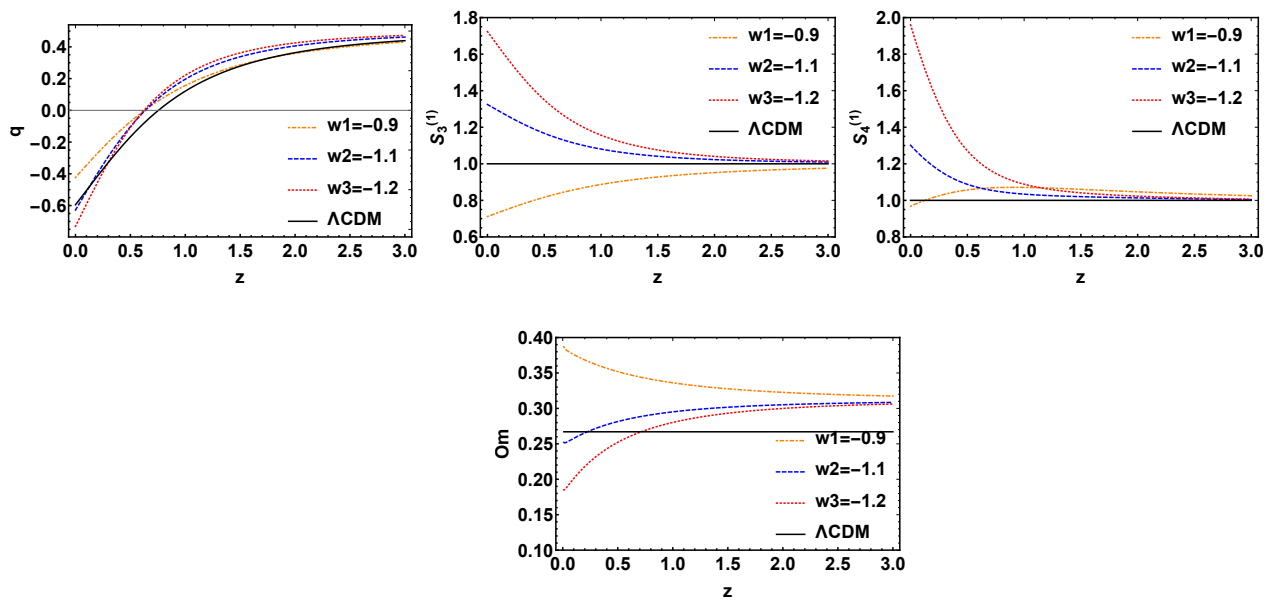

Figure 4. The evolution trajectories of $q, S_{3}^{(1)}, S_{4}^{(1)}$ and $O m$, respectively, versus red shift $\mathrm{z}$ for the $I w C D M 3$ model with variable $w$ and fixed $\xi=0.02$. The evolution curve of $\Lambda C D M$ is also shown in these sets of figures.
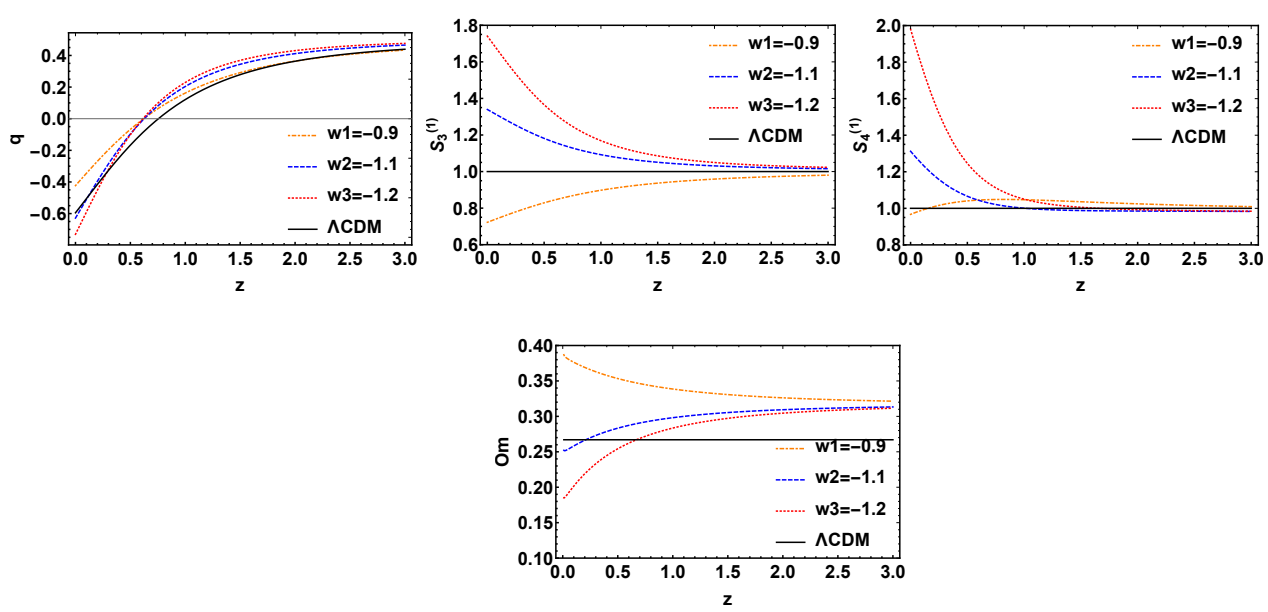

Figure 5. The evolution trajectories of $q, S_{3}^{(1)}, S_{4}^{(1)}$ and $\mathrm{Om}$, respectively, versus red shift $\mathrm{z}$ for the $I w C D M 4$ model with variable $w$ and fixed $\xi=0.02$. The evolution curve of $\Lambda C D M$ is also shown in these sets of figures.
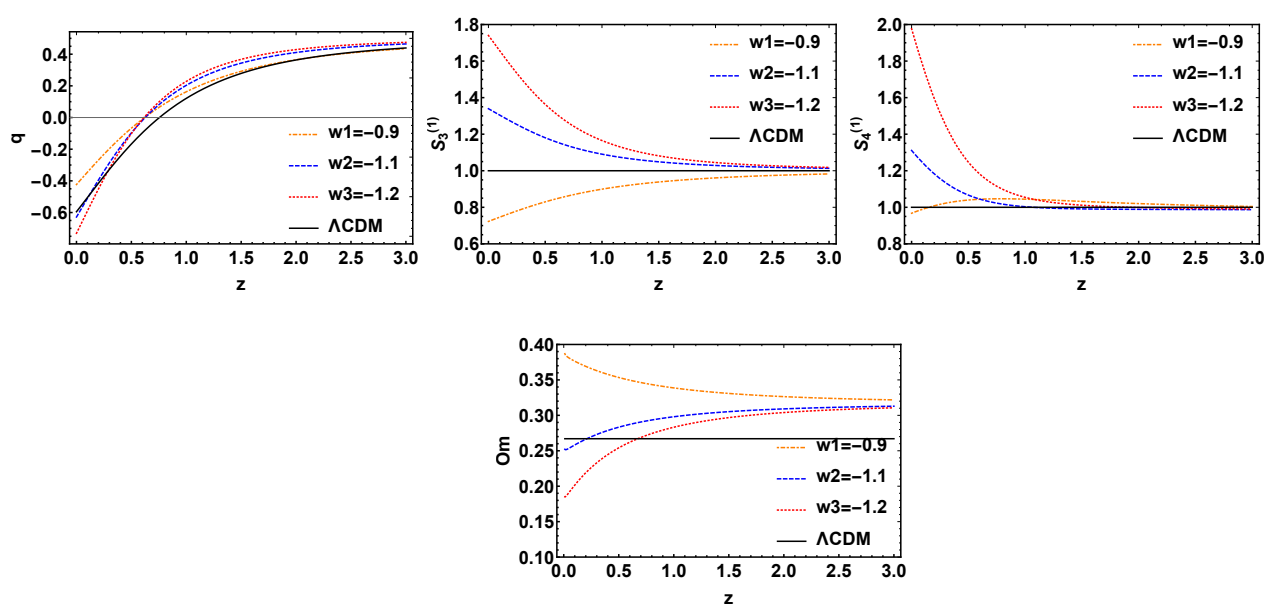

Figure 6. The evolution trajectories of $q, S_{3}^{(1)}, S_{4}^{(1)}$ and $O m$, respectively, versus red shift $\mathrm{z}$ for the I $w C D M 5$ model with variable $w$ and fixed $\xi=0.02$. The evolution curve of $\Lambda C D M$ is also shown in these sets of figures. 

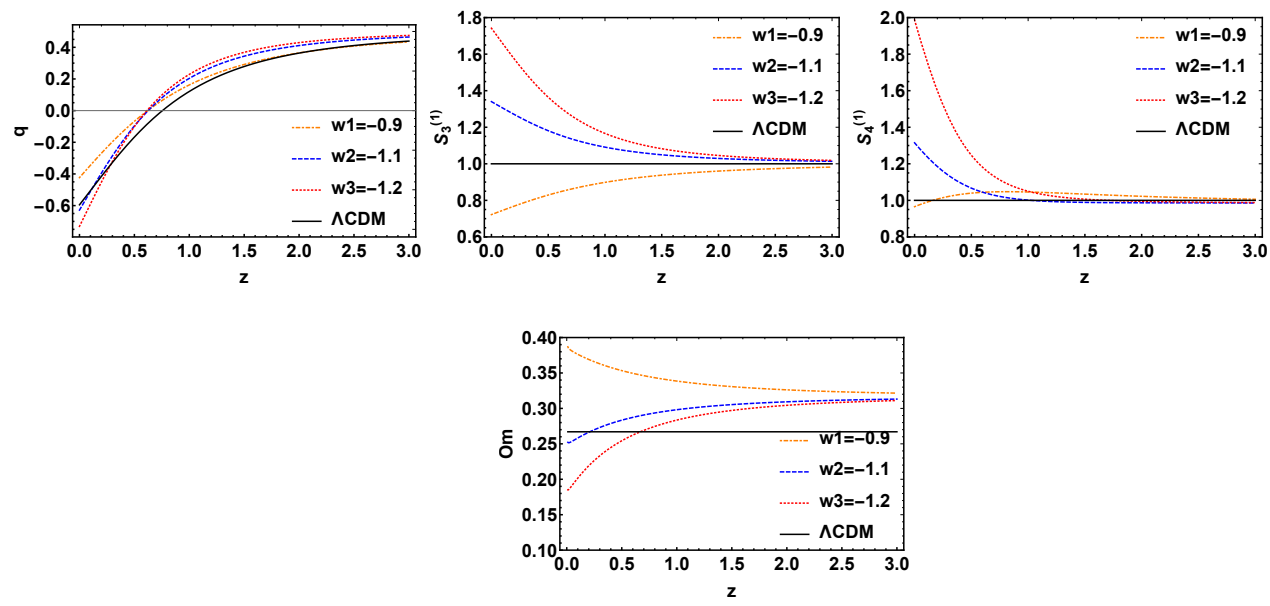

Figure 7. The evolution trajectories of $q, S_{3}^{(1)}, S_{4}^{(1)}$ and $\mathrm{Om}$, respectively, versus red shift $\mathrm{z}$ for the I $w C D M 6$ model with variable $w$ and fixed $\xi=0.02$. The evolution curve of $\Lambda C D M$ is also shown in these sets of figures.

\subsection{Consider the Change of $\xi$ and Keep the Parameter $w$ Constant}

In this subsection, it is only considered that the parameter $\xi$ to take different values on the interaction dark energy model, and keeps the EoS parameter $w$ unchanged.

First of all, we have plotted four diagnostic images of $q, O m, S_{3}^{(1)}$ and $S_{4}^{(1)}$ for the IwCDM1 model in Figure 8. It can be seen that the evolution trajectories in $q$ are not affected by the change of the coupling parameter $\xi$. For $S_{3}^{(1)}$, the evolution trajectories are highly coincident in the high red shift region, and in the low red shift region, although the curves are separated from each other, there is still a high degeneracy. However, $S_{3}^{(1)}$ diagnosis can distinguish $\Lambda C D M$ from IwCDM1 model well in the low red shift region. For $S_{4}^{(1)}$, the evolution is also highly degenerate in the high red shift region, but in the low red shift zone, this degeneracy is destroyed well. In the same way, $S_{4}^{(1)}$ diagnosis can similarly distinguish $\Lambda C D M$ from IwCDM1 model well in the low red shift region. On the contrary, For the $\mathrm{Om}$, the evolution trajectories is highly coincident in the low red shift region, but separated from each other in the high red shift region. In addition, the observations are mainly concentrated in the low red shift range $(z \leq 1)$, so we usually only consider the situation in the low red shift region. In other word, that diagnostic effect of $\mathrm{Om}$ is not good at low red shift region. To sum up, $\mathrm{Om}, \mathrm{S}_{3}^{(1)}$ and $S_{4}^{(1)}$ can all be used to distinguish $\Lambda C D M$ from IwCDM1 model in the low red shift region. However, only $S_{4}^{(1)}$ can distinguish the different evolutionary curves of the IwCDM1 model with different coupling parameter $\xi$ well.

Then, the I $w C D M 2$ model should be discussed now. For Figure 9, it can be found that the evolution curves of $q$ are less affected when the parameter $\xi$ is changed, also it can not be distinguished from $\Lambda C D M$. And the evolution curves of $O m, S_{3}^{(1)}$ and $S_{4}^{(1)}$ for IwCDM2 model have some degeneracy in the low red shift region. But in the high red shift region, they are more separated from each other. However, this is not helpful because of the observations are mainly concentrated in the low red shift range. By comparing these three kinds of diagnosis, we have seen that variable $\xi$ have a greater effect on the evolution curves of $S_{4}^{(1)}$. In other words, the diagnostic effect of $S_{4}^{(1)}$ on the IwCDM2 model is better.

Next we discuss the IwCDM3 model. In Figure 10, it can be seen that the evolution curves of $q$ coincide with each other, and the evolution curves of $\mathrm{Om}$ and $S_{3}^{(1)}$ have high degeneracy in the low red shift. Interestingly, the evolutionary trajectory in $S_{4}^{(1)}$ is completely coincident within $z<0.5$, but when the red shift $z>0.5$, the degeneracy of the curves in $S_{4}^{(1)}$ is broken well, the evolution curves also can be distinguished easily from each other. In general, $S_{4}^{(1)}$ has better diagnostic behavior. 
Then, the three models with interaction terms $Q_{4}, Q_{5}$ and $Q_{6}$ will be discussed, which are called IwCDM4, I $w C D M 5$, I $w C D M 6$. From Figures 11-13, we can see that the evolutionary trajectory of this three models are almost unaffected by the change of parameter $\xi$. So for $O m, S_{3}^{(1)}$ and $S_{4}^{(1)}$, they can only distinguish $\Lambda C D M$ from the IwCDM1 model, but cannot distinguish the evolution curves of variable $\xi$.

In summary, the influence of the state parameter $w$ on the IwCDM model is much greater than that of the coupling parameter $\xi$ on the IwCDM model. $S_{3}^{(1)}$ is a better diagnostic tool when the state parameter $w$ changes and the coupling parameter $\xi$ is fixed, while $S_{4}^{(1)}$ works better when the coupling parameter $\xi$ changes and the state parameter $w$ is unchanged.
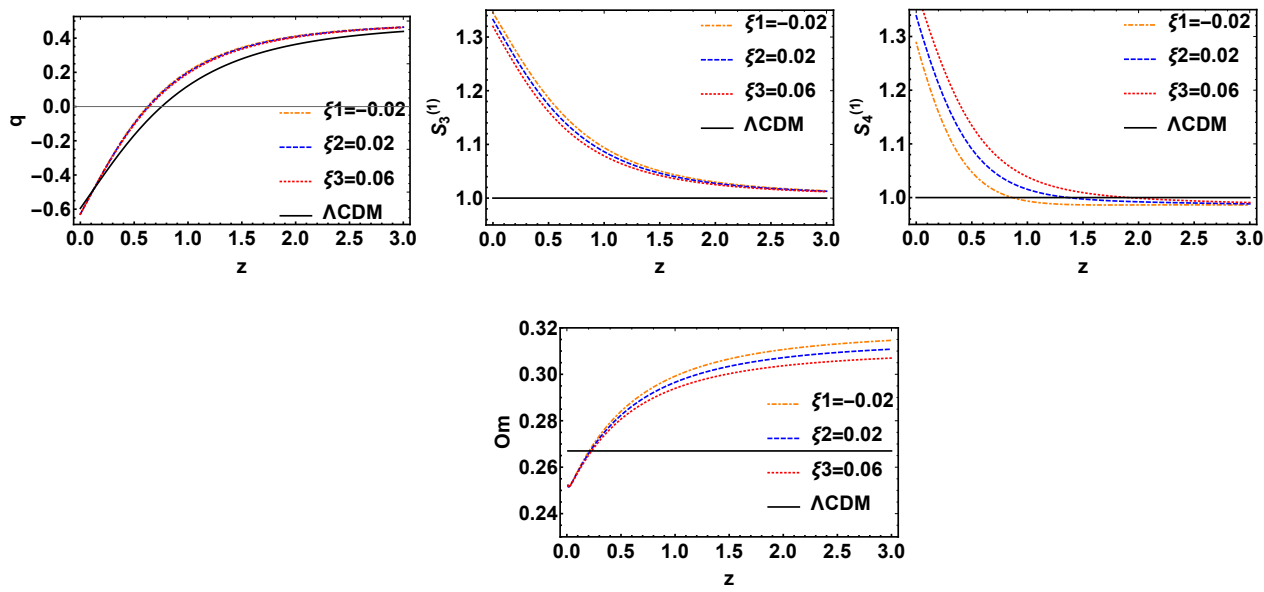

Figure 8. The evolution trajectories of $q, S_{3}^{(1)}, S_{4}^{(1)}$ and $O m$, respectively, versus red shift $\mathrm{z}$ for the I $w C D M 1$ model with variable $\xi$ and fixed $w=-1.1$, where $\xi$ is taken separately $-0.02,0.02,0.06$. The evolution curve of $\Lambda C D M$ is also shown in these sets of figures.
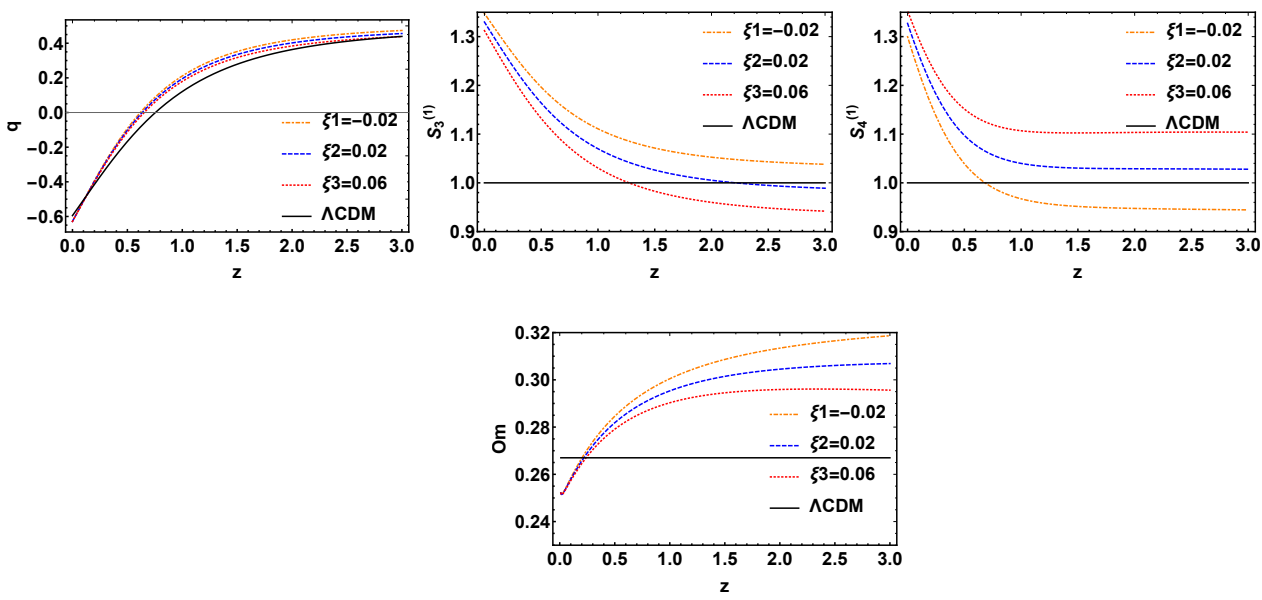

Figure 9. The evolution trajectories of $q, S_{3}^{(1)}, S_{4}^{(1)}$ and $O m$, respectively, versus red shift $\mathrm{z}$ for the I $w C D M 2$ model with variable $\xi$ and fixed $w=-1.1$, where $\xi$ is taken separately $-0.02,0.02,0.06$. The evolution curve of $\Lambda C D M$ is also shown in these sets of figures. 

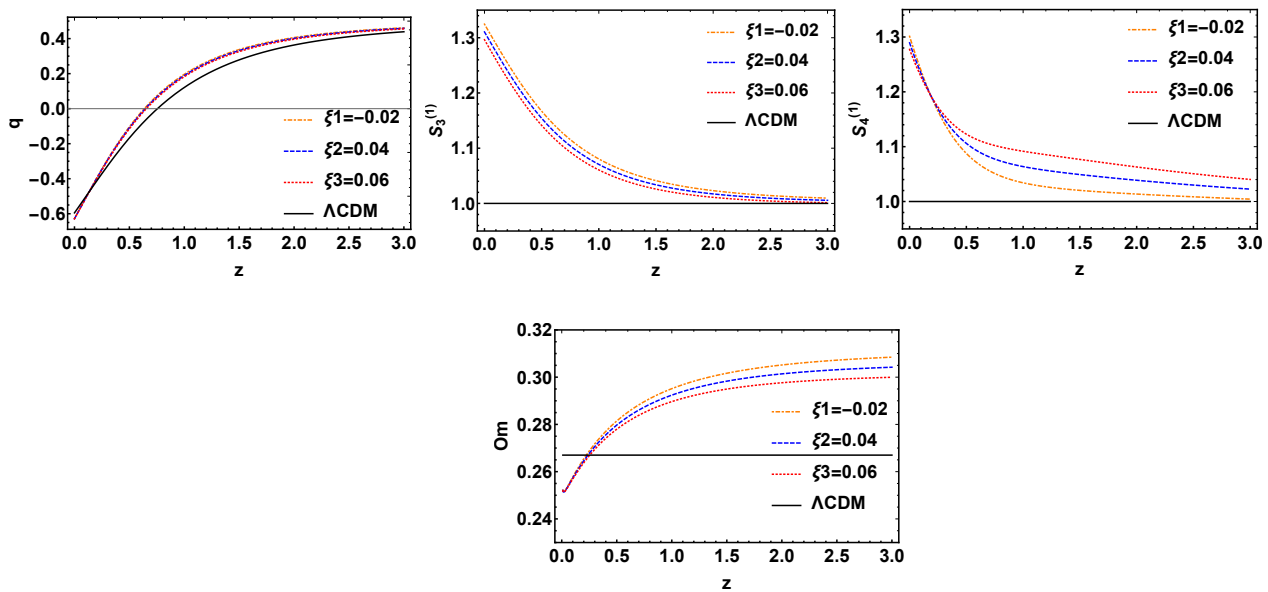

Figure 10. The evolution trajectories of $q, S_{3}^{(1)}, S_{4}^{(1)}$ and $O m$, respectively, versus red shift $\mathrm{z}$ for the I $w C D M 3$ model with variable $\xi$ and fixed $w=-1.1$, where $\xi$ is taken separately $0.02,0.04,0.06$. The evolution curve of $\Lambda C D M$ is also shown in these sets of figures.
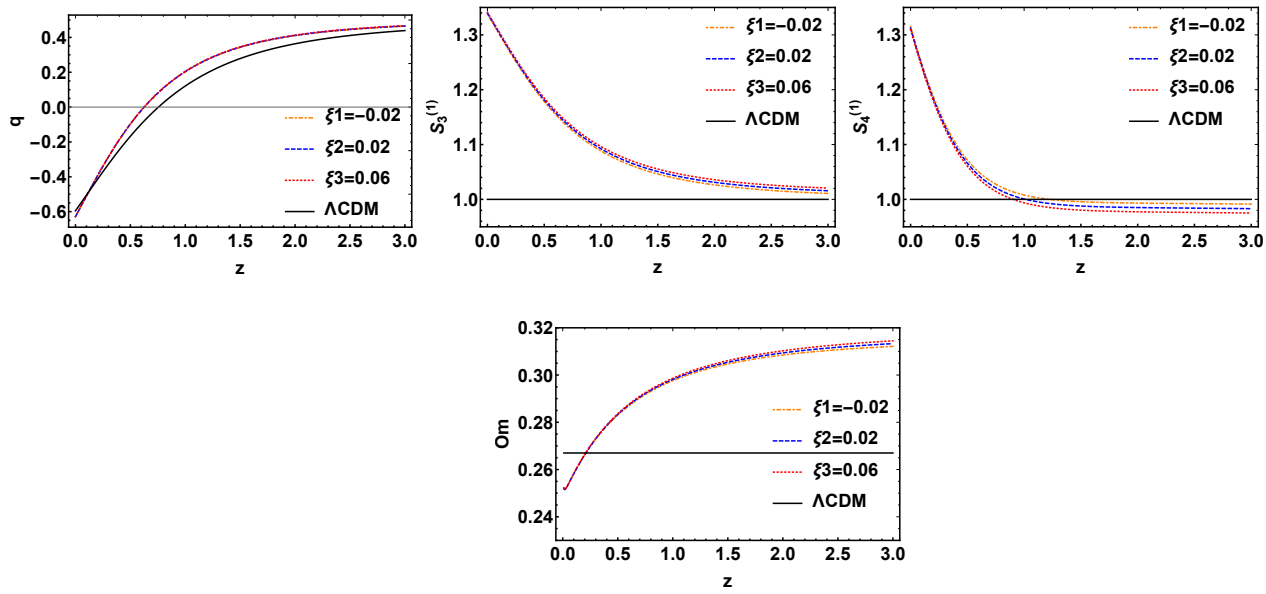

Figure 11. The evolution trajectories of $q, S_{3}^{(1)}, S_{4}^{(1)}$ and $O m$, respectively, versus red shift $\mathrm{z}$ for the I $w C D M 4$ model with variable $\xi$ and fixed $w=-1.1$, where $\xi$ is taken separately $-0.02,0.02,0.06$. The evolution curve of $\Lambda C D M$ is also shown in these sets of figures.
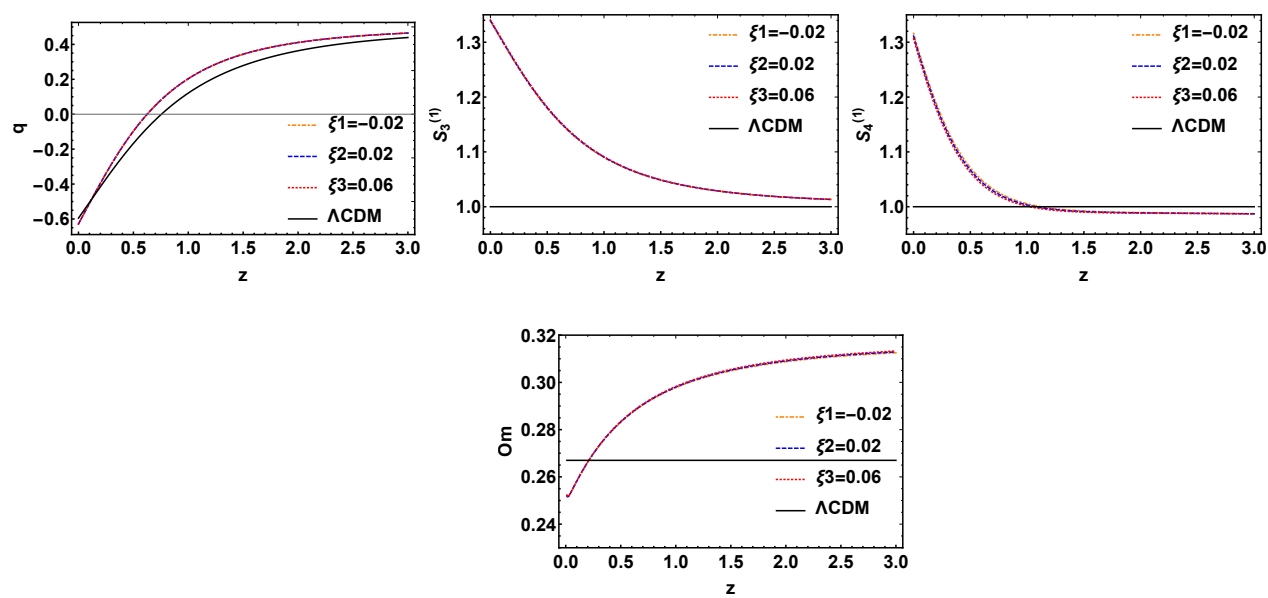

Figure 12. The evolution trajectories of $q, S_{3}^{(1)}, S_{4}^{(1)}$ and $O m$, respectively, versus red shift $\mathrm{z}$ for the IwCDM5 model with variable $\xi$ and fixed $w=-1.1$, where $\xi$ is taken separately $-0.02,0.02,0.06$. The evolution curve of $\Lambda C D M$ is also shown in these sets of figures. 

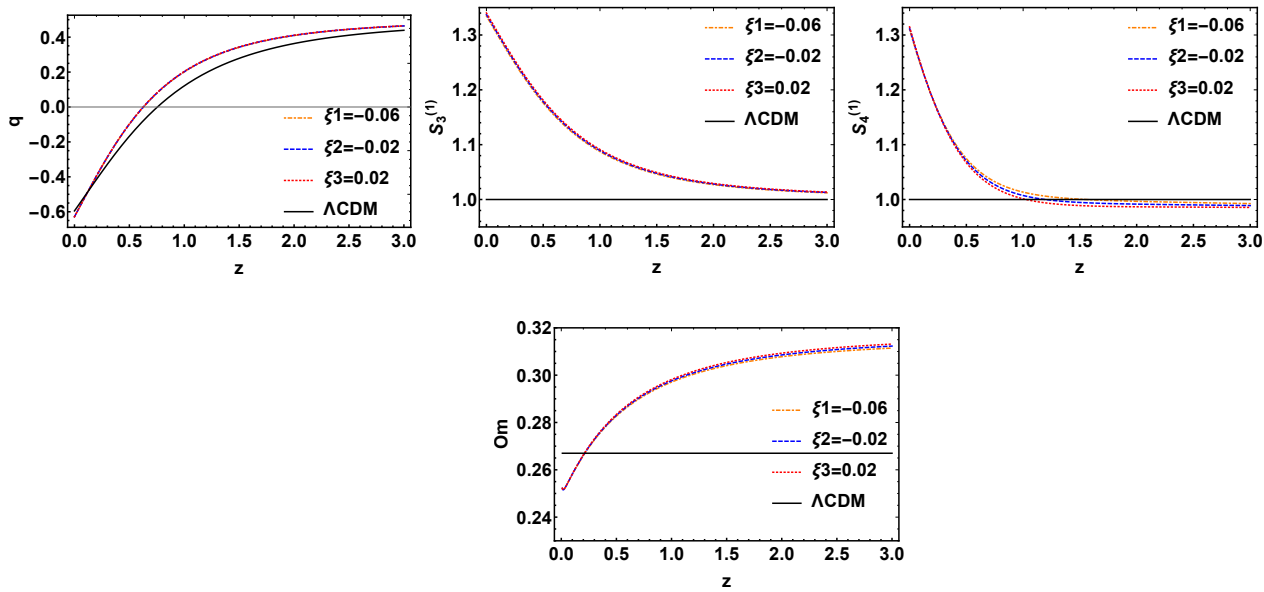

Figure 13. The evolution trajectories of $q, S_{3}^{(1)}, S_{4}^{(1)}$ and $O m$, respectively, versus red shift $\mathrm{z}$ for the I $w C D M 6$ model with variable $\xi$ and fixed $w=-1.1$, where $\xi$ is taken separately $-0.02,0.02,-0.06$. The evolution curve of $\Lambda C D M$ is also shown in these sets of figures.

\section{Conclusions}

In this paper, we have studied the interaction of dark energy models with deceleration parameter $q$, state finder hierarchy and the $O m$ diagnostic. We hope to explore the deviations from $\Lambda C D M$ and to distinguish the evolution trajectory of the interaction dark energy model with different parameters. First, we only take the EoS parameter $w$ with different values on the interaction dark energy model, and kept the coupling parameter $\xi$ constant. We have found that for $q_{(z)}$, the evolution trajectories of different parameters in the high red shift region, is highly degenerate. In the low red shift region, the degeneracy is destroyed to some extent. From $q$, we have seen that the universe is indeed a process from decelerated expansion to accelerated expansion, and no matter how the state parameter $w$ changes, it will not affect the transition red shift. In addition, $\mathrm{Om}, S_{3}^{(1)}$ and $S_{4}^{(1)}$ are good diagnostic methods to distinguish $\Lambda C D M$ from the IwCDM model, and to separate the evolution curves of variable parameter $w$ especially in the low red shift region. We just need to consider the low red shift region because of the observational data are mainly within it. Through the above research, we have found that the evolution curve of the IwCDM model with different values of state parameter $w$ is more separated in $S_{3}^{(1)}$. That is to say, $S_{3}^{(1)}$ is a better tool for diagnostic models. Next, we have only considered the impact of the coupling parameter $\xi$ to the interaction dark energy model, and kept $w$ unchanged. It can be seen that the law of $q$ is similar to that of the previous one. Compared with $\mathrm{Om}, S_{3}^{(1)}$ and $S_{4}^{(1)}$, the evolution curves in $S_{4}^{(1)}$ are more separated from each other. So the diagnosis of $S_{4}^{(1)}$ is better. It should be noted that the change of state parameter $w$ has a greater influence on the $I w C D M$ model than the coupling parameter $\xi$.

As a consequence of all that studied above, we have found that the higher-order statefinder does not diagnose the model as well. We need to make a specific analysis of the specific model.

Since we still know little about the microscopic nature of dark energy and dark matter, it is difficult to calculate a very clear interaction term from a theoretical point of view. Therefore, we can only construct some reasonable interaction terms by phenomenological description, and then make further analysis on this basis. Nowadays, the most widely studied interaction phenomenological model is divided into three main categories, namely, assuming that $Q$ is proportional to dark energy density, $Q$ to dark matter density and $Q$ to the density sum of dark energy and dark matter. The novelty of this paper is that in the construction of the interaction term, we also assume that the $Q$ is proportional to the $\frac{\rho_{c} \rho_{d e}}{\rho_{c}+\rho_{d e}}$, proportional to the $\sqrt{\rho_{c} \rho_{d e}}$, and so on. The study of interaction models is more advantageous than $\Lambda C D M$, and the introduction of interaction between dark matter and dark energy can help us to alleviate or solve some theoretical problems in cosmology, such as the problem of cosmic coincidence, 
the problem of cosmic "great tear" caused by ghost dark energy, and the problem of cosmic age caused by ancient quasars.

Author Contributions: Y.J.: search the references, plot the figures, write the manuscript; Z.H.: plot the figures, design the research plan, data analysis; Q.Z.: data collection, data analysis, edit the manuscript; W.Y.: design the research plan, numerical simulation, revise the manuscript; Y.W.: corresponding author, review the manuscript; revise the manuscript; J.L.: explain the data, data analysis, numerical simulation; H.L.: plot the figures, search the references, edit the manuscript; C.Z.: search the references, arrange the references, design the research plan; Y.W.: explain the data, data analysis, revise the manuscript. All authors have read and agreed to the published version of the manuscript.

Funding: NSFC No. 11575075, 11705079, 11647153.

Acknowledgments: Y. Wu has been supported by the National Natural Science Foundation of China under Grant No. 11575075. W. Yang has been supported by the National Natural Science Foundation of China under Grants No. 11705079 and No. 11647153

Conflicts of Interest: There is no conflict of interest.

\section{References}

1. Iorio, L. Editorial for the Special Issue 100 Years of Chronogeometrodynamics: The Status of the Einstein's Theory of Gravitation in Its Centennial Year. Universe 2015, 1, 38-81. [CrossRef]

2. Debono, I.; Smoot, G.F. General Relativity and Cosmology: Unsolved Questions and Future Directions. Universe 2016, 2, 23. [CrossRef]

3. Riess, A.G. Supernova search team collaboration. Astron. J. 1998, 116, 1009. [CrossRef]

4. Perlmutter, S. Supernova cosmology project collaboration. Astrophys. J. 1999, 517, 565. [CrossRef]

5. Spergel, D.N.; Bean, R.; Dore, O.; Nolta, M.R.; Bennett, C.L.; Dunkley, J.; Hinshaw, G.; Jarosik, N.; Komatsu, E.; Page, L.; et al. WMAP collaboration. Astrophys. J. Suppl. 2007, 170, 377. [CrossRef]

6. Adelman-McCarthy, J.K.; Agueros, M.A.; Allam, S.S.; Prieto, C.A.; Anderson, K.S.J.; Anderson, S.F.; Annis, J.; Bahcall, N.A.; Bailer-Jones, C.A.L.; Baldry, I.K.; et al. SDSS collaboration. Astrophys. J. Suppl. 2008, 175, 297.

7. Tegmark, M.; Strauss, M.; Blanton, M.; Abazajian, K.; Dodelson, S.; Sandvik, H.; Wang, X.; Weinberg, D.; Zehavi, I.; Bahcall, N.; et al. Cosmological parameters from SDSS and WMAP. Phys. Rev. D 2004, 69, 103501. [CrossRef]

8. Ade, P.A.R.; Aghanim, N.; Arnaud, M.; Ashdown, M.; Aumont, J.; Baccigalupi, C.; Banday, A.J.; Barreiro, R.B.; Bartlett, J.G.; Bartolo, N.; et al. Planck Collaboration. Planck 2015 results-XIII. Cosmological parameters. $A \mathcal{E} A$ 2016, 594, A13.

9. Singh, C.P.; Kumar, A. Ricci dark energy model with bulk viscosity. Eur. Phys. J. Plus 2018, 133, 312. [CrossRef]

10. Wang, F.Y.; Dai, Z.G.; Qi, S. Probing the cosmographic parameters to distinguish between dark energy and modified gravity models. Astron. Astrophys. 2009 507, 53-59. [CrossRef]

11. Batista, C.E.; Daouda, M.H.; Fabris, J.C.; Piattella, O.F.; Rodrigues, D.C. Rastall Cosmology and the $\Lambda$ CDM Model. Phys. Rev. D 2012, 85, 084008. [CrossRef]

12. Cao, S.L.; Duan, X.W.; Meng, X.L.; Zhang, T.J. Cosmological model-independent test of $\Lambda$ CDM with two-point diagnostic by the observational Hubble parameter data. Eur. Phys. J. C 2018, 78, 313. [CrossRef]

13. Yang, R.-J.; Zhang, S-N. The age problem in the $\Lambda$ CDM model. Mon. Not. R. Astron. Soc. 2010, 407, 1835. [CrossRef]

14. Popolo, A.D.; Delliou, M.L. Small Scale Problems of the $\Lambda$ CDM Model: A Short Review. Galaxies 2017, 5, 17. [CrossRef]

15. Peebles, P.J.E.; Vilenkin, A. Quintessential inflation. Phys. Rev. D 1999, 59, 063505. [CrossRef]

16. Wang, L.; Steinhardt, P.J. Cluster Abundance Constraints on Quintessence Models. Astrophys. J. 1998, $508,483$. [CrossRef]

17. Caldwell, R.R. A Phantom Menace, Cosmological consequences of a dark energy component with super-negative equation of state. Phys. Lett. B 2002, 545, 23-29. [CrossRef]

18. Guo, Z.K.; Piao, Y.S.; Zhang, X.; Zhang, Y.Z. Cosmological Evolution of a Quintom Model of Dark Energy. Phys. Lett. B 2005, 608, 177-182. [CrossRef] 
19. Zhang, J.; Zhang, X.; Liu, H. Reconstructing generalized ghost condensate model with dynamical dark energy parametrizations and observational datasets. Mod. Phys. Lett. A 2008, 23, 139-152. [CrossRef]

20. Li, M. A Model of Holographic Dark Energy. Phys. Lett. B 2004, 603, 1-5. [CrossRef]

21. Zhang, X.; Wu, F.-Q. Constraints on holographic dark energy from type Ia supernova observations. Phys. Rev. D 2005, 72, 043524. [CrossRef]

22. Zhang, X. Statefinder diagnostic for holographic dark energy model. Int. J. Mod. Phys. D 2005, 14, 1597-1606. [CrossRef]

23. Yu, F.; Zhang, J.-F. Statefinder diagnosis for the extended holographic Ricci dark energy model without and with interaction. Commun. Theor. Phys. 2013, 59, 243.

24. Cui, J.L.; Zhang, J.F. Comparing holographic dark energy models with statefinder. Eur. Phys. J. C 2014, 74, 2849. [CrossRef]

25. Bento, M.C.; Bertolami, O.; Sen, A.A. Generalized Chaplygin gas, accelerated expansion, and dark-energy-matter unification. Phys. Rev. D 2002, 66, 043507. [CrossRef]

26. Bento, M.C.; Bertolami, O.; Sen, A.A. Revival of the unified dark energy—Dark matter model? Phys. Rev. D 2004, 70, 083519. [CrossRef]

27. Kamenshchik, A.; Moschella, U.; Pasquier, V. An alternative to quintessence. Phys. Lett. B 2001, 511, $265-268$. [CrossRef]

28. Garousi, M.R.; Sami, M.; Tsujikawa, S. Constraints on Dirac-Born-Infeld type dark energy models from varying alpha. Phys. Rev. D 2005, 71, 083005. [CrossRef]

29. Cai, R.G.; Wang, S.J. Dark matter superfluid and DBI dark energy. Phys. Rev. D 2016, 93, 023515. [CrossRef]

30. Yang, W.; Pan, S.; Nunes, R.C.; Mota, D.F. Dark calling Dark: Interaction in the dark sector in presence of neutrino properties after Planck CMB final release. arXiv 2019, arXiv:1910.08821.

31. Pan, S.; Yang, W.; Valentino, E.D.; Saridakis, E.N.; Chakraborty, S. Interacting scenarios with dynamical dark energy: Observational constraints and alleviation of the $H_{0}$ tension. Phys. Rev. D 2019, 100, 103520. [CrossRef]

32. Yang, W.; Mena, O.; Pan, S.; Valentino, E.D. Dark sectors with dynamical coupling. Phys. Rev. D 2019, 100, 083509. [CrossRef]

33. Yang, W.; Vagnozzi, S.; Valentino, E.D.; Nunes, R.C.; Pan, S.; Mota, D.F. Listening to the sound of dark sector interactions with gravitational wave standard sirens. JCAP 2019, 1907, 037. [CrossRef]

34. Pan, S.; Yang, W.; Singha, C.; Saridakis, E.N. Observational constraints on sign-changeable interaction models and alleviation of the $H_{0}$ tension. Phys. Rev. D 2019, 100, 083539. [CrossRef]

35. Yang, W.; Banerjee, N.; Paliathanasis, A.; Pan, S. Reconstructing the dark matter and dark energy interaction scenarios from observations. Phys. Dark Univ. 2019, 26, 100383. [CrossRef]

36. Yang, W.; Mukherjee, A.; Valentino, E.D.; Pan, S. Interacting dark energy with time varying equation of state and the $H_{0}$ tension. Phys. Rev. D 2018, 98, 123527. [CrossRef]

37. Yang, W.; Pan, S.; Herrera, R.; Chakraborty, S. Large-scale (in) stability analysis of an exactly solved coupled dark-energy model. Phys. Rev. D 2018, 98, 043517. [CrossRef]

38. Yang, W.; Pan, S.; Valentino, E.D.; Nunes, R.C.; Vagnozzi, S.; Mota, D.F. Tale of stable interacting dark energy, observational signatures, and the $H_{0}$ tension. JCAP 2018, 1809, 019. [CrossRef]

39. Yang, W.; Pan, S.; Barrow, J.D. Large-scale Stability and Astronomical Constraints for Coupled Dark-Energy Models. Phys. Rev. D 2018, 97, 043529. [CrossRef]

40. Yang, W.; Xu, L. Cosmological constraints on interacting dark energy with redshift-space distortion after Planck data. Phys. Rev. D 2014, 89, 083517. [CrossRef]

41. Yang, W.; Xu, L. Testing coupled dark energy with large scale structure observation. JCAP 2014, $1408,034$. [CrossRef]

42. Nunes, R.C.; Pan, S.; Saridakis, E.N. New constraints on interacting dark energy from cosmic chronometers. Phys. Rev. D 2016, 94, 023508. [CrossRef]

43. Sharov, G.S.; Bhattacharya, S.; Pan, S.; Nunes, R.C.; Chakraborty, S. A new interacting two fluid model and its consequences. Mon. Not. R. Astron. Soc. 2017, 466, 3497-3506. [CrossRef]

44. Pan, S.; Mukherjee, A.; Banerjee, N. Astronomical bounds on a cosmological model allowing a general interaction in the dark sector. Mon. Not. R. Astron. Soc. 2018, 477, 1189-1205. [CrossRef]

45. Pan, S.; Bhattacharya, S.; Chakraborty, S. An analytic model for interacting dark energy and its observational constraints. Mon. Not. R. Astron. Soc. 2015, 452, 3038-3046. [CrossRef] 
46. Feng, L.; Li, H.L.; Zhang, J.F.; Zhang, X. Exploring neutrino mass and mass hierarchy in interacting dark energy models. Sci. China Phys. Mech. Astron. 2020, 63, 220401. [CrossRef]

47. Guo, R.Y.; Zhang, J.F.; Zhang, X. Exploring neutrino mass and mass hierarchy in the scenario of vacuum energy interacting with cold dark matte. Chin. Phys. C 2018, 42, 095103. [CrossRef]

48. Feng, L.; Zhang, J.F.; Zhang, X. Search for sterile neutrinos in a universe of vacuum energy interacting with cold dark matter. Phys. Dark Univ. 2019, 23, 100261. [CrossRef]

49. Zhang, J.; An, R.; Luo, W.; Li, Z.; Liao, S.; Wang, B. The First Constraint from SDSS Galaxy-Galaxy Weak Lensing Measurements on Interacting Dark Energy Models. Astrophys. J. 2019, 875, L11. [CrossRef]

50. Zhang, J.; An, R.; Liao, S.; Luo, W.; Li, Z.; Wang, B. Fully self-consistent cosmological simulation pipeline for interacting dark energy models. Phys. Rev. D 2018, 98, 103530. [CrossRef]

51. Shenavar, H.; Javidan, K. A Modified Dynamical Model of Cosmology I Theory. Universe 2020, 6, 1. [CrossRef]

52. Dymnikova, I.; Dobosz, A.; Soltysek, B. Lemaitre class dark energy model for relaxing cosmological constant. Universe 2017, 3, 39. [CrossRef]

53. Deng, X.-M. A Modified Generalized Chaplygin Gas as the Unified Dark Matter-Dark Energy Revisited. Braz. J. Phys. 2011, 41, 333-348. [CrossRef]

54. Bhadra, J.; Debnath, U. Dynamical system analysis of interacting variable modified Chaplygin gas model in FRW universe. Eur. Phys. J. Plus 2012, 127, 30. [CrossRef]

55. Pourhassan, B. Viscous modified cosmic Chaplygin gas cosmology. Int. J. Mod. Phys. 2013, $22,1350061$. [CrossRef]

56. Cui, J.L.; Yin, L.; Wang, L.F.; Li, Y.H.; Zhang, X. A closer look at interacting dark energy with statefinder hierarchy and growth rate of structure. JCAP 2015, 09, 024. [CrossRef]

57. Zhang, Z.; Li, S.; Li, Xi.; Zhang, X.; Li, M. Revisit of the Interaction between Holographic Dark Energy and Dark Matter. JCAP 2012, 06,009 . [CrossRef]

58. Wang, B.; Gong, Y.; Abdalla, E. Transition of the dark energy equation of state in an interacting holographic dark energy model. Phys. Lett. B 2005, 624, 141-146. [CrossRef]

59. Hu, B.; Ling, Y. Interacting dark energy, holographic principle and coincidence problem. Phys. Rev. D 2006, 73, 123510. [CrossRef]

60. Setare, M.R.; Vagenas, E.C. The cosmological dynamics of interacting holograhic dark energy model. Int. J. Mod. Phys. 2009, 18, 147-157. [CrossRef]

61. Cao, S.; Liang, N. Interacting between dark energy and dark matter: Observational conditions from OHD, BAO, CMB AND SNe Ia. Int. J. Mod. Phys. D 2013, 22, 1350082. [CrossRef]

62. Wang, J.S.; Wang, F.Y. Cosmological model of the interaction between dark matter and dark energy. $A \mathcal{E} A$ 2014, 564, A137.

63. He, J.-H.; Wang, B. The interaction between dark energy and dark matter. J. Phys. Conf. Ser. 2010, $222,012029$. [CrossRef]

64. Tamanini, N. On phenomenological models of dark energy interacting with dark matter. Phys. Rev. D 2015, 92, 043524. [CrossRef]

65. Wei, H.; Cai, R.-G. Statefinder Diagnostic and w-w' Analysis for the Agegraphic Dark Energy Models without and with Interaction. Phys. Lett. B 2007, 655, 1-6. [CrossRef]

66. Arabsalmani, M.; Sahni, V. The Statefinder hierarchy: An extended null diagnostic for concordance cosmology. Phys. Rev. D 2011, 83, 043501. [CrossRef]

67. Zhang, J.-F.; Cui, J.-L.; Zhang, X. Diagnosing holographic dark energy models with statefinder hierarchy. Eur. Phys. J. C 2014, 74, 3100. [CrossRef]

68. Yu, F.; Cui, J.-L.; Zhang, J.-F.; Zhang, X. Statefinder hierarchy exploration of the extended Ricci dark energy. Eur. Phys. J. C 2015, 75, 274. [CrossRef]

69. Zhao, Z.; Wang, S. Diagnosing holographic type dark energy models with the Statefinder hierarchy, composite null diagnostic and $\mathrm{w}-\mathrm{w}^{\prime}$ pair. Sci. China Phys. Mech. Astron. 2018, 61, 039811. [CrossRef]

70. Zhou, L.; Wang, S. Diagnosing $\Lambda$ HDE model with statefinder hierarchy and fractional growth parameter. Sci. China Phys. Mech. Astron. 2016, 59, 670411. [CrossRef]

71. Li, S.; Ma, Y.; Chen, Y. Dynamical Evolution of Interacting Modified Chaplygin Gas. Int. J. Mod. Phys. D 2009, 18, 1785-1800. [CrossRef] 
72. Khurshudyan, M.Z.; Makarenko, A.N. On a phenomenology of the accelerated expansion with a varying ghost dark energy. Astrophys. Space Sci. 2016, 361, 187. [CrossRef]

73. Chang, B.; Liu, H.; Xu, L.; Zhang, C. Statefinder Parameters for Five-Dimensional Cosmology. Mod. Phys. Lett. A 2008, 23, 269-279. [CrossRef]

74. Alam, U.; Sahni, V.; Saini, T.D.; Starobinsky, A.A. Exploring the Expanding Universe and Dark Energy using the Statefinder Diagnostic. Mon. Not. R. Astron. Soc. 2003, 344, 1057-1074. [CrossRef]

75. Sahni, V.; Shafieloo, A.; Starobinsky, A.A. Two new diagnostics of dark energy. Phys. Rev. D 2008, 78, 103502. [CrossRef]

76. Shafieloo, A.; Sahni, V.; Starobinsky, A.A. A new null diagnostic customized for reconstructing the properties of dark energy from BAO data. Phys. Rev. D 2012, 86, 103527. [CrossRef]

77. Sahni, V.; Shafieloo, A.; Starobinsky, A.A. Model independent evidence for dark energy evolution from Baryon Acoustic Oscillations. Astrophys. J. 2014, 793, 40. [CrossRef]

78. Malekjani, M.; Khodam-Mohammadi, A.; Nazari-pooya, N. Cosmological evolution and statefinder diagnostic for new holographic dark energy model in non flat universe. Astrophys. Space Sci. 2011, 332, 515-524. [CrossRef]

(C) 2020 by the authors. Licensee MDPI, Basel, Switzerland. This article is an open access article distributed under the terms and conditions of the Creative Commons Attribution (CC BY) license (http://creativecommons.org/licenses/by/4.0/). 